\title{
Monoclonal Antibody T101 F(ab)2
}

National Cancer Institute

\section{Source}

National Cancer Institute. Monoclonal Antibody T101 F(ab)2. NCI Thesaurus. Code C29481.

A synthetic mouse monoclonal antibody which targets CD5+ lymphocytes. Monoclonal antibody T101 may be used in the immunotherapy of T-cell malignancies. Containing only the antigen-binding fragment of an immunoglobulin molecule, an FAB (fragment antibody) offers the advantages of smaller size and lower cross-reactivity compared to complete antibodies. ( $\mathrm{NCl04)}$ 